\title{
Colaborações em Pesquisas sobre Educação à Distância: uma Análise de Redes Sociais de Autores
}

\author{
Rodrigo Richard Gomes - richard@ pucminas.br - PUC Minas \\ Marcelo Werneck Barbosa - mwerneck@ pucminas.br-PUC Minas
}

Resumo. Muitas pesquisas, inclusive as realizadas na área de Educação à Distância (EaD), são feitas por meio de colaborações entre pesquisadores. Colaborações por meio de co-autoria formam uma rede na qual os nodos representam autores e uma ligação entre eles existe se eles publicaram um trabalho juntos. Tal rede de colaboração é um tipo de rede social estudada e caracterizada pelo método de Análise de Redes Sociais (ARS). O objetivo deste trabalho foi caracterizar a comunidade mundial de pesquisa em EaD utilizando ARS para identificar os pesquisadores mais centrais e importantes desta área. Uma caracterização da rede de colaborações em nível macro e micro foi realizada.

Palavras-chave: Educação à Distância, Análise de Redes Sociais, MOOCs.

\section{Collaborations on Distance Education Researches: A Social Network Analysis of Authors}

\begin{abstract}
A lot of research, including studies in Distance Education, is performed through collaborations among researchers. Collaborations by means of co-authorship form a network in which nodes represent authors and a connection exists between them if they have published a paper together. This collaboration network is a kind of social network which is studied and characterized by the Social Network Analysis (SNA) method. This work aims at characterizing the world research community of Distance Education using SNA in order to identify those researchers who are more central and important in this area. A characterization of the collaboration network in macro and micro level was performed.
\end{abstract}

Keywords: Distance Education, Social Network Analysis, MOOCs.

\section{Introdução}

A educação vem passando por uma fase expressiva de mudanças. Nesse sentido, as inovações tecnológicas assumem uma posição cada vez mais importante. A Educação à Distância $(\mathrm{EaD})$ é uma modalidade, amplamente usada nos processos de ensinoaprendizagem (Bremgartner, Netto e Menezes, 2015), que tem buscado conciliar o uso das tecnologias ao processo educacional, com o intuito de ampliar as possibilidades educacionais. A EaD pode ser definida como o processo de ensino-aprendizagem, mediado por tecnologias, no qual professores e alunos estão separados espacial ou temporalmente (Brenner et al., 2014).

$\mathrm{O}$ desenvolvimento da $\mathrm{EaD}$ se deu em três gerações, iniciando com os estudos por correspondência; posteriormente com as mídias eletrônicas e finalmente com os MOOCs (Massive Open Online Course ou Curso Online Aberto e Massivo). Desta 
forma, MOOCs podem ser vistos como uma extensão da EaD (Vaidya e Paranjape, 2014). MOOCs, como o nome sugere, são iniciativas de grande escala na prestação de cursos on-line. Eles se desenvolveram a partir do aumento da especialização das universidades no uso do ensino à distância e recursos educacionais abertos. A EaD como indústria vem crescendo; no entanto, os problemas muitas vezes associados a ela são a qualidade limitada dos recursos e materiais para a aprendizagem on-line, a interação dos estudantes e acadêmicos e dificuldades relacionadas às avaliações. Os MOOCs trouxeram avanços que incluem o envolvimento dos melhores acadêmicos nas principais universidades internacionais, o desenvolvimento de materiais de aprendizagem de excelente qualidade e a oferta de cursos gratuitos (Clarke, 2013). O número de cursos abertos, incluindo os MOOCs, está crescendo (Olsson, 2016). Atualmente, há ao menos 8 milhões de estudantes em cursos MOOC em todo o mundo e vários milhares de cursos sendo oferecidos (Porter, 2015).

A evolução da $\mathrm{EaD}$ tem sido alvo de muitos estudos (Brenner et al., 2014). Apesar de as origens da pesquisa em EaD estarem nos anos 30, somente agora ela está demonstrando a maturidade requerida para o fenômeno completo e multifacetado da $\mathrm{EaD}$ (Saba, 2014). Anderson e Zawacki-Richter (2014) afirmam que há uma natureza variada de temas e pesquisadores e contextos divergentes na pesquisa educacional. Tal variedade e riqueza de temas são observadas no trabalho de Liyanagunawardena, Adams e Williams (2013), que apresenta uma revisão sistemática das pesquisas sobre MOOCs realizadas entre os anos de 2008 e 2012. Julgando ser o primeiro estudo com esta temática, os autores analisaram 45 artigos e dividiram os estudos em 8 categorias relacionadas aos métodos e objetivos de pesquisa. Os autores concluem que o uso dos MOOCs gera uma enorme quantidade de dados que não tem sido amplamente analisada, restringindo nossa compreensão sobre os mesmos. Barin e Bastos (2013), por meio de um levantamento e análise da produção bibliográfica, realizaram uma análise de 16 trabalhos científicos sobre MOOCs com o objetivo de identificar potencialidades e oportunidades destes cursos.

Muitas pesquisas, inclusive as realizadas na área de $\mathrm{EaD}$, são feitas por meio de colaborações entre pesquisadores. O trabalho de Zawacki-Richtera e von Prümmera (2010) realizou um estudo bibliométrico das colaborações em pesquisas em EaD com foco nas relações de gênero. O estudo considerou uma amostra de 695 artigos publicados em 5 grandes periódicos da área entre os anos de 2000 e 2008. O trabalho observou uma tendência de aumento de colaborações na área, sendo que pesquisadoras do sexo feminino tendem a escolher temas mais diversificados quando comparadas aos pesquisadores do sexo masculino. Há ainda uma concentração dos pesquisadores em áreas estereotipadas em relação ao sexo - homens pesquisam mais temas relacionados à tecnologia enquanto mulheres trabalham com assuntos relacionados à comunicação e características dos alunos.

Colaborações são naturais e intrínsecas a redes de pesquisadores. Existem diversas formas de colaboração, tais como as que acontecem por meio de projetos de pesquisa, visitas a universidades, co-citação e co-autoria (Hu e Racherla, 2010; Ye e Law, 2013), sendo as duas últimas as formas mais utilizadas para investigar comunidades de pesquisa. A análise de co-citações, apesar de ser um método popular de análise, não captura os aspectos sociais que apoiam a transmissão de ideias científicas nem como as comunidades de pesquisa são formadas. Por sua vez, a análise de coautorias permite a construção de medidas observáveis e visuais de uma comunidade. 
Além disso, co-autoria é o tipo de informação mais frequentemente usado para explicar padrões de comportamento entre pesquisadores (Stefano, Giordano e Vitale, 2011). A importância crescente de publicação de pesquisas na academia aliada aos avanços na tecnologia da informação proporcionaram um aumento de co-autorias em áreas diferentes (Behara, Sunil e Smart, 2014; Cainelli, Maggioni, Uberti e Felice, 2015).

Colaborações por meio de co-autoria formam uma rede na qual os nodos da rede representam autores e uma conexão entre eles existe se dois autores publicaram um trabalho juntos. Tal rede de colaboração é um tipo de rede social. O estudo destas redes, seus participantes e interações entre eles é chamado de Análise de Redes Sociais (ARS) ou Social Network Analysis (SNA) (Wasserman e Faust, 1994). O uso de ARS tem crescido em função do desenvolvimento de técnicas de visualização e computação (Sloane e O'Reilly, 2013) e se tornou uma das técnicas de análise interdisciplinar mais populares (Ye e Law, 2013).

Neste contexto, o objetivo deste trabalho é caracterizar a comunidade mundial de pesquisa em EaD utilizando Análise de Redes Sociais para identificar os pesquisadores mais centrais e importantes desta área. Este trabalho contribui para os estudos da área no sentido que se desconhecem estudos desta natureza no campo de pesquisa em EaD além do realizado por Zawacki-Richtera e von Prümmera (2010). Entretanto, o estudo referenciado é um estudo bibliométrico, com foco em gênero e limitado a um período relativamente curto. Nosso estudo é abrangente e com foco em mapear toda a pesquisa neste campo.

O restante deste artigo está organizado da seguinte forma. A Seção 2 apresenta o referencial teórico cobrindo os aspectos da técnica de Análise de Redes Sociais. A Seção 3 descreve a metodologia utilizada neste estudo enquanto a Seção 4 apresenta as análises das métricas oriundas do estudo da rede de autores. A Seção 5 apresenta conclusões do trabalho, suas limitações e possibilidades de trabalhos futuros.

\section{Análise de Redes Sociais}

Indivíduos e organizações interagem entre si de diversas maneiras. Para facilitar o estudo de tais interações, redes têm sido utilizadas para modelar e representar graficamente estas interações e os atores que participam das mesmas. Na representação visual de uma rede, os nodos (ou nós) são representados por pontos ou círculos e as relações entre eles são desenhadas como linhas que conectam dois ou mais nodos.

Outra forma de representar esta rede é por meio de uma matriz de adjacências, na qual linhas e colunas representam os nodos da rede e a interseção entre colunas é preenchida com um número 0 se não há relação entre os objetos e o número 1 se há uma relação entre eles ou até mesmo um número que represente a intensidade desta relação. Em tais redes, os vértices representam pessoas e organizações e as linhas representam interação, informação, comunicação, amizade, troca de bens ou fluxos (Behara et al., 2014).

A Análise de Redes Sociais é uma metodologia para estudar redes de comunicação informais. Este tipo de comunicação acontece quando pessoas discutem ideias em várias lugares como locais de trabalho e conferências, quando engajados em relacionamentos como orientador/orientado, colegas e co-autores (Marion et al., 2003). Um dos usos mais importantes da ARS é a identificação dos autores que são mais 
centrais dentro da rede. Centralidade é o atributo estrutural das relações entre atores em uma rede em vez de ser um atributo específico de tais atores (Carter, Ellram e Tate, 2007). Centralidade em uma rede social é um conceito que ilustra os mais importantes atores na rede. Atores com alta centralidade possuem uma localização estratégica dentro da rede. Há muitas maneiras de medir as dimensões da importância de um ator na rede. Uma forma possível é a de calcular seu grau (degree) de centralidade, que define o nó mais importante como aquele com o maior número de ligações. Grau de centralidade representa o número total de nodos aos quais um nó específico está ligado. É a medida mais comum e mais simples de avaliar o grau de colaboração (Ye e Law, 2013).

Outra possível métrica de centralidade é a centralidade de intermedialidade do nó (node-betweenness centrality). Ela refere-se ao grau em que um ponto particular situa-se entre os vários outros nodos no gráfo: um nó de um grau relativamente baixo pode desempenhar um papel importante intermediário e assim ser muito central para a rede. Assim nodos que estão "entre" outros podem agir como conexões na disseminação de conhecimento entre a rede (Giannakis, 2012). A centralidade de intermedialidade refere-se ao número de caminhos que passam por um ator sobre os caminhos mais curtos que ligam dois outros atores. $\mathrm{O}$ conceito por trás dessa métrica é baseado no fluxo de informações: um nó tem alta centralidade de intermedialidade quando se encontra em muitos caminhos mais curtos que ligam dois outros nodos. Esta é uma métrica importante, uma vez que um nó com alta centralidade de intermedialidade tem melhor acesso à informação, está mais bem posicionado para agir como intermediário nas trocas e pode ser capaz de controlar os fluxos de comunicação e pode potencialmente servir como um elo entre áreas isoladas da rede (Sloane e O 'Reilly, 2013). Em termos de intercâmbios acadêmicos e fluxo de conhecimento, os pesquisadores que têm alta centralidade de intermedialidade estão no núcleo da rede de colaboração (Ye e Law, 2013).

\section{Procedimentos Metodológicos}

Este estudo é classificado como exploratório-descritivo, pois o seu objetivo é ampliar o conhecimento a respeito de um determinado fenômeno, explorar a realidade e buscar maior conhecimento sobre um tema para depois detalhar os fatos descobertos. A abordagem adotada foi a qualitativa, que se concentra no aprofundamento dos valores contidos nos dados. Esse tipo de análise tem por base conhecimentos teóricos e empíricos que permitem atribuir-lhe cientificidade (Zanella, 2009).

Para elaboração da rede de pesquisadores em EaD, foi necessário identificar os estudos por meio dos quais os autores e suas colaborações seriam avaliados. Foi então realizada uma busca na base científica Web of Science. Esta base foi escolhida por ser relevante no contexto científico e ter sido utilizada em estudos similares. Para obtenção das publicações, de acordo com o referencial teórico estudado, foi realizada pesquisa pelas palavras-chave: "online learning" OR "MOOC" OR "distance education" OR "massive open online course", pois esta string de busca representa os temas de interesse desta pesquisa. Foram encontradas por esta pesquisa 2947 publicações. Decidiu-se por manter apenas os trabalhos publicados em periódicos (journals), de forma a se obter uma rede de publicações de alta qualidade. Por fim, foram retirados os artigos cujos nomes dos autores são escritos em outro alfabeto, como o coreano. Restaram então 
1532 artigos para a análise. A rede estudada foi montada com base neste conjunto de artigos.

\section{Resultados}

\subsection{Indicadores do conjunto de artigos}

Para se formar a rede de colaborações a ser estudada, foram considerados como atores da rede apenas os autores em artigos que apresentaram co-autoria. A Tabela 1 apresenta alguns dados gerais sobre o conjunto de artigos avaliado.

Tabela 1. Dados Gerais da Amostra. Fonte (dados da pesquisa)

\begin{tabular}{|c|c|c|c|c|c|c|c|}
\hline \multicolumn{5}{|c|}{ Total de artigos identificados } & & & 1532 \\
\hline \multicolumn{5}{|c|}{ Artigos com um autor } & & & 710 \\
\hline \multicolumn{5}{|c|}{ Artigos com colaboração } & & & 822 \\
\hline \multicolumn{5}{|c|}{ Artigos com dois autores } & & & 379 \\
\hline \multicolumn{5}{|c|}{ Artigos com três autores } & & & 205 \\
\hline \multicolumn{5}{|c|}{ Artigos com quatro autores ou mais } & & & 238 \\
\hline \multicolumn{5}{|c|}{ Total de autores } & & & 3116 \\
\hline \multicolumn{5}{|c|}{ Total de co-autores } & & & 2505 \\
\hline \multicolumn{5}{|c|}{ Total de colaborações } & & & 4407 \\
\hline \multicolumn{8}{|c|}{ Artigos publicados por período } \\
\hline Período & $\mathrm{N}^{\mathrm{o}}$ artigos & Período & $\mathrm{N}^{\mathrm{o}}$ artigos & Período & $\mathrm{N}^{\mathrm{o}}$ artigos & Período & $\mathrm{N}^{\mathrm{o}}$ artigos \\
\hline $\begin{array}{c}1977- \\
1980\end{array}$ & 8 & $\begin{array}{c}1981- \\
1985\end{array}$ & 29 & $\begin{array}{c}1986- \\
1990\end{array}$ & 79 & $\begin{array}{c}1991- \\
1995\end{array}$ & 100 \\
\hline $\begin{array}{l}1996- \\
2000\end{array}$ & 172 & $\begin{array}{l}2001- \\
2005\end{array}$ & 239 & $\begin{array}{c}2006- \\
2010\end{array}$ & 326 & $\begin{array}{l}2011- \\
2016\end{array}$ & 581 \\
\hline
\end{tabular}

Artigos com um único autor representaram 46,3\% do conjunto de artigos avaliados, enquanto os artigos que apresentam co-autoria representam 53,7\% do universo pesquisado. Esse dado é similar ao trabalho de Ye e Law (2013) sobre redes de colaborações de pesquisa em Turismo no qual o índice de artigos com apenas um autor foi de 40,5\%. Do total de autores únicos do universo de artigos pesquisados, foi possível observar que $80,3 \%$ dos autores publicaram algum trabalho em colaboração com outros autores e apenas 19,6\% publicaram artigos sem colaboração. Para cada par de autores que participaram como co-autores de um trabalho foi considerada uma colaboração. No total, 4407 colaborações foram identificadas nesta rede. Entre os artigos que apresentaram colaboração, os escritos por dois autores representaram $46,1 \%$, os escritos por 3 autores representaram $24,9 \%$ enquanto os escritos por 4 ou mais autores totalizaram $29 \%$ dos artigos com colaboração. É possível observar ainda na Tabela 1 uma evolução das publicações na área ao longo dos anos. Considerando o período de publicações entre 1977 e 2016, mais de um terço do total de artigos foi publicado nos últimos 5 anos. 
O estudo das redes considerando a técnica de ARS é baseado em algumas propriedades destas redes definidas em dois níveis: macro e micro, apresentados respectivamente nas próximas seções.

\subsection{Características Macro da Rede}

Algumas características globais da rede ajudam a explicar como se forma a rede de colaborações em $\mathrm{EaD}$. A rede obtida neste estudo apresentou um grau médio igual a 3,419. Essa medida indica que autores que publicam artigos na área de EaD têm, em média, 3,4 colaboradores. A densidade de uma rede é definida como a proporção de conexões existentes na rede dentre todas as conexões possíveis, logo, é um valor definido entre 0 e 1 . No contexto de uma rede de co-autorias, esta métrica representa a porcentagem da rede com a qual um autor publicou um trabalho (Hu e Racherla, 2010). A rede obtida é extremamente esparsa, apresentando densidade de 0,001. Considerando que a rede estudada envolve colaborações entre autores de todo o mundo, é esperado e consistente com outros trabalhos que a densidade seja realmente baixa.

Outra característica macro importante é o diâmetro. O diâmetro de uma rede é considerado a maior distância entre dois participantes da rede e representa o esforço de se atravessar a rede. Neste estudo, a rede apresentou um diâmetro de 6, indicando que atravessando 6 conexões se pode chegar a qualquer autor da rede. Este resultado é um indício do fenômeno denominado "seis graus de separação" característico de redes chamadas "small world". Neste tipo de rede, a maioria dos nodos não são vizinhos uns dos outros, mas estão separados por poucas conexões (Cainelli et al., 2015). Tal resultado é corroborado pela distância média obtida de 1,098. Este valor mostra que os autores estão em média próximos uns aos outros.

\subsection{Características Micro da Rede}

Esta seção apresenta as características e métricas relacionadas aos nodos da rede, ou seja, em nível micro. A primeira métrica a ser analisada é o grau de centralidade, que indica o número de conexões que cada nó da rede possui. Em uma rede de co-autorias, este número reflete o número de colaborações realizadas por cada pesquisador. Uma variante do grau de centralidade é o índice de Bonacich. Esta métrica representa o quão inserido na rede um autor está. Ela considera os nodos da rede que estão ligados aos vizinhos de cada nó. Assim, a centralidade de um nó depende não somente das conexões que cada nó possui, mas também das conexões de seus vizinhos. Se um autor se conecta a vários autores com altos graus de centralidade, então se considera que este autor está em uma posição central na rede quando comparado a nodos mais isolados.

A Tabela 2 apresenta os valores obtidos para o Grau de Centralidade e Índice de Bonacich para os 8 pesquisadores mais centrais da rede. $\mathrm{Na}$ rede, não ocorreram divergências entre o posicionamento dos autores quanto aos dois índices. 
Tabela 2. Classificação de autores por grau e índice Bonacich

\begin{tabular}{|l|l|l|}
\hline Autor & Grau & Índice de Bonacich \\
\hline Bernard, Robert M. & 17 & 30,577 \\
\hline Borokhovski, Eugene & 16 & 29,639 \\
\hline Aggarwal, Surabhi & 15 & 26,986 \\
\hline Dunn, Carolyn & 15 & 26,986 \\
\hline Nordby, Kelly & 15 & 26,986 \\
\hline Thomas, Cathy & 15 & 26,986 \\
\hline Abrami, Philip C. & 14 & 25,869 \\
\hline Kember, D & 13 & 25,084 \\
\hline
\end{tabular}

Foi também objetivo desta pesquisa identificar os autores que mais publicaram trabalhos e os que mais participaram de colaborações. A Tabela 3 apresenta estes dados para os 10 autores com mais publicações. Pode-se observar que o número de publicações não apresenta uma relação direta com o número de colaborações, ou seja, há autores que não publicaram tantos trabalhos, mas apresentam muitas colaborações.

Tabela 3. Número de publicações e colaborações dos principais autores

\begin{tabular}{|c|c|c|}
\hline Autor & Número de publicações & Número de colaborações \\
\hline Richardson, John T. E. & 8 & 12 \\
\hline Bernard, Robert M. & 5 & 17 \\
\hline Anderson, Terry & 5 & 10 \\
\hline Richardson, JTE & 5 & 6 \\
\hline Borokhovski, Eugene & 4 & 16 \\
\hline Kember, D & 4 & 13 \\
\hline Jelfs, Anne & 4 & 6 \\
\hline Ghaffari, Azam & 4 & 4 \\
\hline Abrami, Philip C. & 3 & 14 \\
\hline Tamim, Rana M. & 3 & 12 \\
\hline
\end{tabular}

Uma métrica importante para se determinar a centralidade de um autor na rede é a centralidade de intermedialidade. Esta métrica considera se um nó da rede está no menor caminho de muitos pares de nodos e, consequentemente, está em uma posição crítica para agir como distribuidor de informação nesta rede. Em uma rede de coautorias, um pesquisador com alta centralidade de intermedialidade pode ser considerado como alguém que une diversos grupos de pesquisa e publica trabalhos com pesquisadores que não trabalhariam juntos se não fosse por este autor. A Tabela 4 apresenta os valores dos autores com maiores índices de centralidade de intermedialidade. Pode-se observar como esta métrica gera resultados diferentes, pois apenas o pesquisador Bernard, Robert M. consta nas Tabelas 2, 3 e 4. 
Tabela 4. Classificação dos Autores por Centralidade de intermedialidade

\begin{tabular}{|l|r|}
\hline Autor & Centralidade de intermedialidade \\
\hline Anderson, Terry & 52,000 \\
\hline Zawacki-Richter, Olaf & 44,000 \\
\hline Vellenga, B & 27,000 \\
\hline Sigulem, Daniel & 24,000 \\
\hline Latchem, Colin & 23,000 \\
\hline Vogt, Sebastian & 22,000 \\
\hline Block, DE & 20,000 \\
\hline Pritchard, David E. & 20,000 \\
\hline Shaw, Tim & 18,000 \\
\hline Bernard, Robert M. & 13,083 \\
\hline
\end{tabular}

\subsection{Análise de componentes e cliques}

Uma rede pode ser subdividida em componentes e cliques. Um componente é um conjunto de nodos, onde cada nodo possui um caminho para todos os outros nodos do conjunto. A rede obtida apresenta 738 componentes. Cliques, um tipo especial de componente, são definidas como o maior conjunto de nodos no qual todos os nodos estão diretamente relacionados a todos os outros. Assim, em uma rede de colaborações, uma clique é composta por um grupo de autores no qual todos os autores publicaram um trabalho em conjunto com todos os outros participantes da clique.

A rede identificada apresenta 792 cliques com no mínimo 2 autores. Neste estudo, cada artigo publicado por 2 ou mais autores é uma clique, uma vez que todos os autores estão conectados uns aos outros por terem uma relação de colaboração para publicar o artigo. Assim, partindo da premissa que cada publicação se torna uma clique nesta rede, chega-se à conclusão de que alguns autores repetem a colaboração em alguns artigos, uma vez que há 822 artigos, mas apenas 792 cliques. Isso significa que em 30 artigos houve repetição na lista de autores, ou seja, estes autores já haviam colaborado em um artigo anterior.

\section{Conclusões e Trabalhos Futuros}

Este estudo teve como objetivo caracterizar a rede de pesquisadores em EaD no mundo por meio da Análise de Redes Sociais. Com o auxílio desta técnica, foi possível obter características globais e locais desta rede e também identificar quem são os pesquisadores mais centrais desta rede. Entende-se esta caracterização como um trabalho inicial no sentido de fomentar o uso de ARS em pesquisas de EaD. Tal técnica de análise permite interpretações muito ricas, que podem ser exploradas em estudos futuros.

Este estudo apresenta algumas limitações. Apesar de ter-se realizado a busca inicial com o objetivo de identificar amplamente os estudos em $\mathrm{EaD}$, somente foi utilizada uma base de dados científica. Ampliar a pesquisa para considerar outras bases, 
pode incluir mais pesquisadores. No entanto, isso não muda o fato de que o conjunto de dados de artigos analisados compreende pesquisa de qualidade representativa no campo. Além disso, não foram tratados sistematicamente problemas de ambiguidade dos nomes dos autores, algo típico neste tipo de pesquisa.

Este estudo abre várias possibilidades de trabalhos futuros. O estudo se concentrou especificamente sobre a caracterização dessa comunidade de pesquisa em termos de co-autoria. No entanto, existem outras formas de colaboração que foram mantidas fora do nosso alcance, tais como projetos de pesquisa e parcerias. Além disso, este estudo incidiu sobre um retrato atual da comunidade de pesquisa. Estudar sua evolução e também ser capaz de prever futuras colaborações é um nicho de pesquisa interessante. Outra possibilidade seria ainda analisar colaborações em pesquisas de subcampos da EaD, o que permitiria identificar os grupos de pesquisadores mais influentes em cada um deles.

Finalmente, este estudo também usa um extenso conjunto de dados que exige processamento para ser trabalhado. Embora todo esforço tenha sido feito para manter a integridade dos dados, deve-se notar que qualquer erro ou omissão possível é inteiramente acidental.

\section{Referências Bibliográficas}

ANDERSON, T.; ZAWACKI-RICHTER, O. Towards a Research Agenda. Online distance education: Towards a research agenda, 2014. Editora AU Press.

BARIN, C.S.; BASTOS, F.P. Problematização dos MOOC na atualidade: Potencialidades e Desafios. Novas Tecnologias na Educação, v.11, n.3, 2013.

BEHARA, R. S., SUNIL, B., SMART, P. A. Leadership in OM research: a social network analysis of European researchers. International Journal of Operations and Production Management, v.34, n.12, 2014.

BREMGARTNER, V.; NETTO, J. F. M.; MENEZES, C. S. Adaptation Resources in Virtual Learning Environments under Constructivist Approach: A Systematic Review. Frontiers in Education Conference, pp. 1-8, 2015.

BRENNER, F.; PIANESSER, R.; ESPÍNDOLA, D. B.; PINTO, I.; MUSA, M.; GOUVEA, A.; GALLON, R. F.; MACHADO, K. S. Revisão Sistemática da Educação a Distância: Um estudo de Caso da EaD no Brasil. XI Congresso Brasileiro de Ensino Superior a Distância, 2014, Florianópolis, Santa Catarina.

CAINELli, G., MAGGIONI, M. A., UBERTI, T. E., FELICE, A. De. The strength of strong ties: How co-authorship affect productivity of academic economists? Scientometrics, 102(1), pp. 673-699, 2015.

CARTER, C. R.; ELLRAM, L. M.; TATE, W. The use of social network analysis in logistics research. Journal of Business Logistics, Vol. 28, N.1 , pp. 137-168, 2007.

CLARKE, T. The advance of the MOOCs (massive open online courses), Education + Training, Vol. 55 Iss 4/5 pp. 403 - 413, 2013.

GIANNAKIS, M. The intellectual structure of the supply chain management discipline. Journal of Enterprise Information Management, v.25, n.2, pp.136-169, 2012. 
HU, C., RACHERLA, P. A Social Network Perspective of Tourism Research. Annals of Tourism Research, 37(4), pp. 1012-1034, 2010.

LIYANAGUNAWARDENA, T. R.; ADAMS, A. A.; WILLIAMS, S. A. MOOCs: A Systematic Study of the Published Literature 2008-2012. The International Review of Research in Open and Distributed Learning, Vol 14 | No 3, pp. 202-227, 2013.

MARION, L. S., GARFIELD, E., HARGENS, L. L., LIEVROUW, L. A., WHITE, H. D., WILSON, C. S. Social Network Analysis and Citation Network Analysis: Complementary Approaches to the Study of Scientific Communication Sponsored by SIG MET. Proceedings of the American Society for Information Science and Technology, v.40, n.1, pp. 486-487, 2003.

OLSSON, U. Open courses and MOOCs as professional development - is the openness a hindrance? Education + Training, Vol. 58 Iss 2 pp. 229 - 243, 2016.

PORTER, S. The economics of MOOCs: a sustainable future?, The Bottom Line, Vol. 28 Iss $1 / 2$ pp. $52-62,2015$.

SABA, F. Methods of Study in Distance Education: A Critical Review of Selected Recent Literature. Online distance education: Towards a research agenda, 2014. Editores: Zawacki-Richter, O. e Anderson, T. Editora AU Press.

SLOANE, A.; O' REILLY, S. Production Planning \& Control: The Management of Operations The emergence of supply network ecosystems : a social network analysis perspective. Production Planning \& Control, 24(7), pp. 621-639, 2013.

STEFANO, D. De; GIORDANO, G.; VITALE, M. P. Issues in the analysis of coauthorship networks. Quality \& Quantity, 45(5), pp. 1091-1107, 2011.

VAIDYA, S.; PARANJAPE, A. MOOCs - Changing the way of education. IEEE International Conference on MOOC, 2014.

WASSERMAN, S.; FAUST, K. Social Network Analysis: Methods and applications, 1994. Cambridge: Cambridge University Press.

YE, Q., LAW, R. A coauthorship network analysis of tourism and hospitality research. Journal of Hospitality \& Tourism Research, 37(1), pp. 51-76, 2013.

ZANELLA. L. C. H. Metodologia de estudo e de pesquisa em administração, 2009, Florianópolis: Departamento de Ciências da Administração/UFSC.

ZAWACKI-RICHTERA, O.; von PRÜMMERA, C. Gender and collaboration patterns in distance education research. Open Learning: The Journal of Open, Distance and e-Learning. Volume 25, Issue 2, pp. 95-114. 2010 